\title{
The Reliability of an Upper- and Lower-Extremity Visuomotor Reaction Time Task
}

\author{
Caitlin Brinkman, Shelby E. Baez, Carolina Quintana, Morgan L. Andrews, \\ Nick R. Heebner, Matthew C. Hoch, and Johanna M. Hoch
}

\begin{abstract}
Context: Fast visuomotor reaction time (VMRT), the time required to recognize and respond to sequentially appearing visual stimuli, allows an athlete to successfully respond to stimuli during sports participation, while slower VMRT has been associated with increased injury risk. Light-based systems are capable of measuring both upper- and lower-extremity VMRT; however, the reliability of these assessments are not known. Objective: To determine the reliability of an upper- and lower-extremity VMRT task using a light-based trainer system. Design: Reliability study. Setting: Laboratory. Patients (or Other Participants): Twenty participants with no history of injury in the last 12 months. Methods: Participants reported to the laboratory on 2 separate testing sessions separated by 1 week. For both tasks, participants were instructed to extinguish a random sequence of illuminated lightemitting diode disks, which appeared one at a time as quickly as possible. Participants were provided a series of practice trials before completing the test trials. VMRT was calculated as the time in seconds between target hits, where higher VMRT represented slower reaction time. Main Outcome Measures: Separate intraclass correlation coefficients (ICCs) with corresponding 95\% confidence intervals (CIs) were calculated to determine test-retest reliability for each task. The SEM and minimal detectable change values were determined to examine clinical applicability. Results: The right limb lower-extremity reliability was excellent $\left(\mathrm{ICC}_{2,1}=.92 ; 95 \% \mathrm{CI}, .81-.97\right)$. Both the left limb $\left(\mathrm{ICC}_{2,1}=.80 ; 95 \% \mathrm{CI}, .56-.92\right)$ and upper-extremity task $\left(\mathrm{ICC}_{2,1}=.86 ; 95 \%\right.$ CI, .65-.95) had good reliability. Conclusions: Both VMRT tasks had clinically acceptable reliability in a healthy, active population. Future research should explore further applications of these tests as an outcome measure following rehabilitation for health conditions with known VMRT deficits.
\end{abstract}

Keywords: injury risk, light-based trainer system, functional performance

Reaction time is the speed of response to an environmental stimulus. ${ }^{1}$ Types of reaction time include simple reaction time, choice reaction time, and visuomotor reaction time (VMRT). VMRT is the time required to recognize and respond to sequentially appearing visual stimuli. ${ }^{2}$ Previous studies have identified that slower VMRT is associated with increased injury risk. ${ }^{2}$ It could be important for clinicians and sports performance specialists to continue to assess VMRT to identify injury risk and increase performance in an athletic population.

Visuomotor reaction time is often measured using commercial devices. For example, Wilkerson et $\mathrm{al}^{2}$ identified a relationship between upper-extremity VMRT and lower-extremity injury risk with the Dynavision D2 system (Dynavision; Dynavision International LLC, West Chester, OH). The Dynavision D2 system provides a reliable measure of VMRT, 3,4 reliant upon upper-extremity movement strategies; however, this system cannot be configured to assess lower-extremity movement strategies. ${ }^{4,5} \mathrm{~A}$ task that can assess lower-extremity VMRT may provide greater insight into lowerextremity injury risk and potential impairments created by lowerextremity injury. ${ }^{2}$ While systems such as the Dynavision D2 show potential for providing unique insights into lower-extremity injury risk and novel directions for injury prevention, the system's lack of

Brinkman and J.M. Hoch are with the Department of Athletic Training and Clinical Nutrition, University of Kentucky, Lexington, KY, USA. Baez is with Michigan State University, East Lansing, MI, USA. Quintana, Heebner, and M.C. Hoch are with the Sports Medicine Research Institute, University of Kentucky, Lexington, KY, USA. Andrews is with Army-Baylor University, San Antonio, TX, USA. J.M. Hoch (Johanna.hoch@uky.edu) is corresponding author. portability and inability of the participant to move physical location to respond to stimuli are limiting. The ability to test or train VMRT in different environments and create novel test setups which introduce a level of variance into VMRT testing or training may be valuable for integration into clinical practice. Recent technological developments, such as the FitLight system (FITLIGHT Trainer ${ }^{\mathrm{TM}}$ Sports Corp, Canada), may provide more versatile options to measure VMRT, which can easily be configured for upper- and lower-extremity applications. ${ }^{6}$ Therefore, assessing standardized tests designed to assess upper- and lower-extremity VMRT, with a portable device that could be translated to a variety of environments, is warranted.

At this time, there are no data available to support the reliability of an upper- or lower-extremity VMRT task using the FitLight system. For use in the clinic, reliability and consistency measures should be determined. Therefore, the purpose of this study was to determine the test-retest reliability of an upper- and lower-extremity VMRT task using the FitLight system. We hypothesize both tasks will have clinically acceptable test-retest reliability for assessing VMRT in a healthy population.

\section{Methods}

\section{Design}

A test-retest design was used to determine the reliability of an upper- and lower-extremity VMRT task. The independent variable was time (session 1 and session 2) and the dependent variables were the average time between target hits (VMRT, in seconds), upper- and lower-extremity "hits," and upper-extremity "misses." 


\section{Participants}

A total of 20 participants (14 females, age: 24.1 [3.8] y, mass: 68.4 [11.7] kg, height: 159.2 [38.8] cm, 19 right limb dominant) were recruited from the general student population at a large academic institution and surrounding areas. A power analysis revealed 18 participants would be needed to achieve $90 \%$ power if $R_{0}=.7$ and $R_{1}=.9 .^{7}$ Participants were excluded if they self-reported they had a lower-extremity injury in the last 12 months (eg, knee, hip, ankle), were taking any medications known to affect the central or peripheral nervous system, were pregnant or nursing, had been diagnosed with a peripheral neurological condition, had been diagnosed with a concussion in the last 12 months or were symptomatic from a previous concussion at the time of testing, or had been diagnosed with epilepsy or had a history of seizures. This study was reviewed and approved by the University of Kentucky Institutional Review Board, and all participants signed an informed consent.

\section{Procedures}

Participants reported to 2 testing sessions separated by 1 week. After informed consent was obtained, participants completed a demographic health questionnaire. Next, participants completed the upper-extremity task followed by the lower-extremity task. The lower-extremity task is more physically demanding; thus, we opted to complete the lower-extremity task after the completion of the upper-extremity task to decrease the risk of fatigue impacting

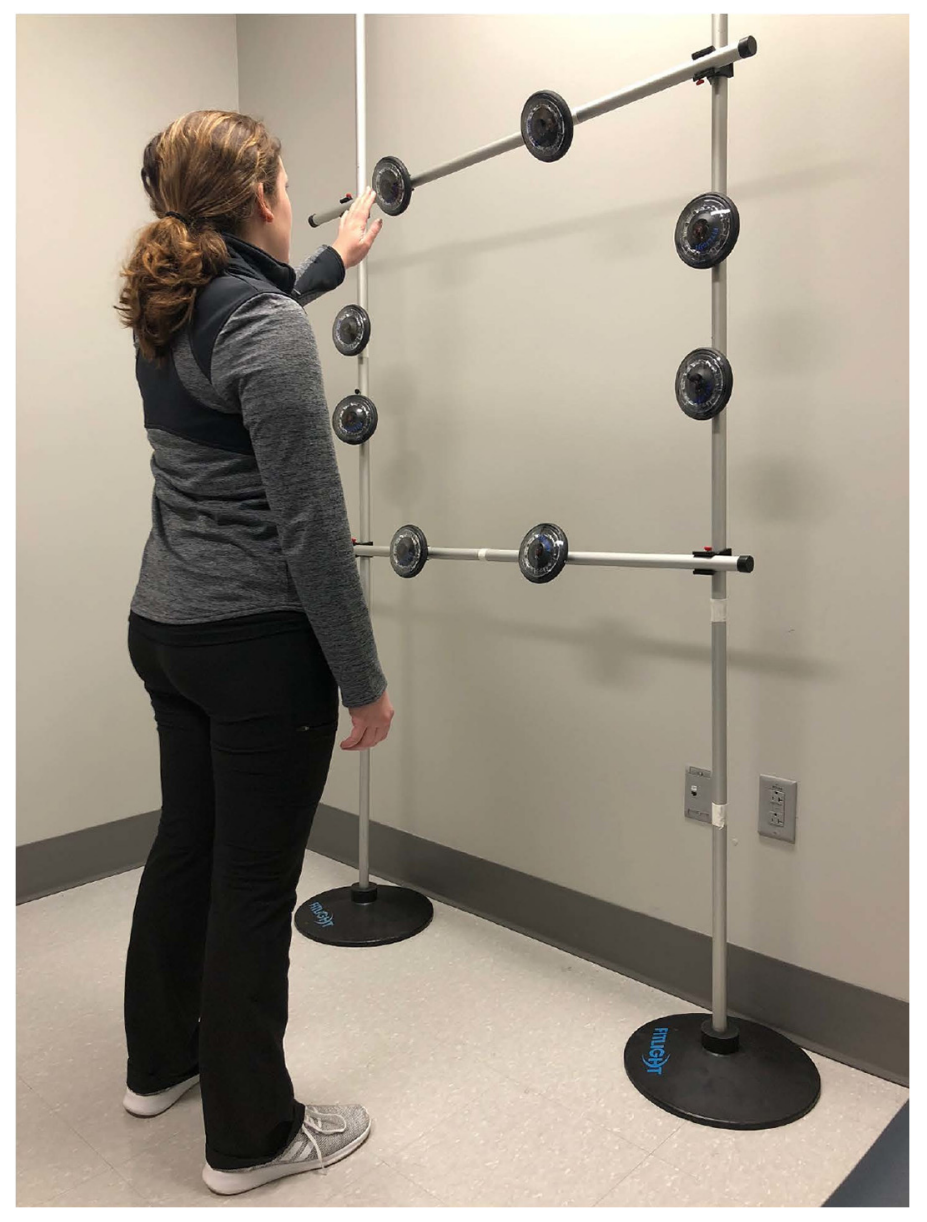

Figure 1 - The upper-extremity visumotor reaction time task. outcomes. The tasks were administered by a group of 5 clinicians, who followed standard operating procedures for each session.

\section{Instrumentation}

The FitLight system (FITLIGHT Trainer ${ }^{\mathrm{TM}}$ Sports Corp) is a speed and agility trainer comprised of light-emitting diode wireless sensor light disks designed to be deactivated by hands, feet, head, and/or sports equipment. The equipment is versatile, which allows the system to be easily implemented in different environments, ${ }^{6}$ such as a laboratory, clinic, or indoor/outdoor sport venues. Deactivation of lights can occur through contact with the target or through proximity of the target. The FitLight system has been previously used to examine VMRT in athletes for sport performance training purposes. ${ }^{8,9}$

For the upper-extremity task, participants were placed in front of a large grid-like formation (Figure 1) and instructed to respond to a visual stimulus and deactivate a series of 8 lights in a random sequence. Positioning was normalized to reach distance by having the participant stand away from the grid at the maximal distance where they were able to reach each sensor. The random sequence of visual stimuli was configured using the hand-eye coordination setting, under performance and training (Table 1). The participants were instructed that they could use both hands to deactivate the lights. The participants had 0.80 seconds to deactivate the target to be counted as a "hit," otherwise the light would extinguish and be counted as a "miss." The time to deactivation was selected because it was the closest this parameter could be set based on other commercially available devices. The participants completed two 30 -second practice trials and three 1-minute test trials.

For the lower-extremity task, participants were instructed to respond to a visual stimulus and deactivate a series of 5 targets arranged in a semicircle with their feet. Participants were placed standing in the front of a $180^{\circ}$ semicircle, with each light disk placed in increments of $45^{\circ}$ around the semicircle. The distance for lights 1 to 4 was normalized to the length of each participant's shank, which was measured as the distance from the tibial tuberosity to the medial malleolus. Light 5 (Figure 2), the light closest to the lateral malleolus of the stance leg, was placed at a distance equal to half the length of the shank. The purpose of normalization was to decrease the emphasis on balance. Participants stood on one limb, while deactivating the targets in front with the moving limb. The random sequence was configured using the hand-eye coordination setting, under performance and training (Table 1). Participants completed three 30 -second practice trials for each leg, followed by

Table 1 FitLight System Tablet Parameters for the Upper- and Lower-Extremity Protocols

\begin{tabular}{lcc}
\hline Parameter & $\begin{array}{c}\text { Upper-extremity } \\
\text { protocol }\end{array}$ & $\begin{array}{c}\text { Lower-extremity } \\
\text { protocol }\end{array}$ \\
\hline Distance & $10 \mathrm{~cm}$ & N/A \\
Impact & N/A & 1 \\
sensitivity & $0.8 \mathrm{~s}$ & Off \\
Timeout & $0.05 \mathrm{~s}$ & $0.05 \mathrm{~s}$ \\
Delay & Center & Full \\
Light mode & Distance & Impact \\
Sensor mode & Off & On \\
Light beep & &
\end{tabular}

Note: N/A, not applicable. 
one 1-minute test trial for each leg. Limb order was counterbalanced, and participants had a 30 -second rest period between trials to decrease the effects of fatigue. The test limb was represented as the limb that was moving.

\section{Statistical Analyses}

Descriptive statistics were calculated for all demographic variables (mean [SD]). Test-retest reliability was determined using intraclass correlation coefficients (ICCs) with corresponding 95\% confidence intervals (CIs). One-rating (single measure), consistency, 2-way mixed-effects model ICCs were conducted for the lower-extremity VMRT and hits. Upper-extremity VMRT and hits and misses reliability were determined using separate mean-rating (3), absolute agreement, 2-way mixed-effects model ICCs. The ICCs were interpreted as poor $(<.50)$, moderate $(.50-.75)$, good (.75-.90), and excellent $(>.90) .{ }^{10}$ For each ICC, the SEM and minimal detectable change $(\mathrm{MDC}=\mathrm{SEM} \times 1.96 \times \sqrt{ } 2)$ at the $95 \%$ confidence $($ MDC95) were calculated. All statistical analyses were performed using SPSS software (version 25.0; SPSS, Inc, Chicago, IL).

\section{Results}

Descriptive statistics, SEM, and MDC values for the upper- and lower-extremity tasks are presented in Table 2. For VMRT, the right limb lower-extremity task had excellent reliability and both the left limb and upper-extremity task had good reliability $(\mathrm{ICCs}=$

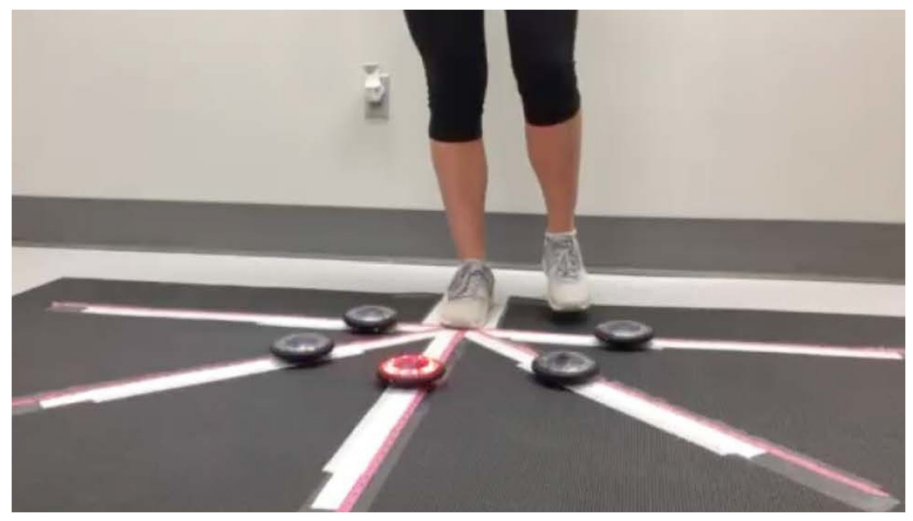

Figure 2 - The lower-extremity visumotor reaction time task.
.80-.96, Table 2). For misses, the upper-extremity task had moderate reliability (Table 2). For hits, the right and left limb lower- and the upper-extremity task had good reliability $(\mathrm{ICCs}=.77-.83$, Table 2).

\section{Discussion}

Both the upper- and lower-extremity VMRT tasks had good-toexcellent reliability in healthy young adults. The upper-extremity task demonstrated moderate reliability for misses, while the upperand lower-extremity tasks demonstrated good-to-excellent reliability for hits. Both tasks demonstrated clinically acceptable reliability for implementation in clinical practice. The Dynavision D2 system upper-extremity VMRT task has been established. ${ }^{3}$ The Dynavision D2 includes 64 raised targets, ${ }^{3}$ while the FitLight system upper-extremity task in this protocol included 8 lights. We concluded the upper-extremity VMRT task using the FitLight system is a reliable assessment. Therefore, clinicians now have 2 reliable mechanisms to assess VMRT in their athletes or patients for training or rehabilitation purposes.

We developed a novel lower-extremity VMRT task using the FitLight system. While the literature has examined the relationship between upper-extremity VMRT and injury risk, ${ }^{2}$ to our knowledge, no previous research has examined the relationship between a lower-extremity VMRT task and injury risk. Participants in Wilkerson et $\mathrm{al}^{2}$ completed three 30-second familiarization trials, followed by one 1-minute test trial. We replicated the number of familiarization and test trials for the lower-extremity task described herein. An additional consideration for the lower-extremity task was the placement of the light disks around the participant. We normalized the distance of the lights to the length of the participant's shank. This normalization was done to control the nature of the task, with the goal to limit testing the participant's balance, and control for variability between participants.

There are numerous applications for both tasks. The FitLight system used for these tasks has been marketed for rehabilitation and performance training purposes. We calculated the MDC95 for the upper-extremity $(0.03 \mathrm{~s})$, and the right $(0.07 \mathrm{~s})$ and left $(0.11 \mathrm{~s})$ lower-extremity tasks. Clinicians could use these tasks to measure the VMRT as an outcome of treatment effectiveness; the MDC95 values can be used to assess clinically meaningful changes in reaction time. Future research should consider examining effective intervention strategies to improve VMRT, using the upper- or lowerextremity protocols described as outcome measures.

Table 2 Descriptive Statistics and Test-Retest Reliability for the Upper- and Lower-Extremity Visuomotor Reaction Time Variables

\begin{tabular}{|c|c|c|c|c|c|}
\hline Task & Session 1 & Session 2 & $\begin{array}{c}\text { ICC } \\
(95 \% \mathrm{CI})\end{array}$ & SEM & $\begin{array}{c}\text { MDC } \\
(95 \% \mathrm{Cl})\end{array}$ \\
\hline UE reaction time, $\mathrm{s}$ & $0.48(0.03)$ & $0.46(0.03)$ & $.86(.65-.95)$ & 0.01 & 0.03 \\
\hline UE misses, $\mathrm{n}$ & $14.6(8.7)$ & $11.3(7.7)$ & $.73(.31-.89)$ & 4.30 & 11.9 \\
\hline UE hits, $\mathrm{n}$ & $82.2(16.7)$ & $88.9(16.9)$ & $.77 .41-.91)$ & 8.15 & 22.6 \\
\hline Left LE reaction time, $s$ & $0.58(0.1)$ & $0.54(0.1)$ & $.80(.56-.92)$ & 0.09 & 0.11 \\
\hline Left LE hits, $\mathrm{n}$ & $90.5(11.9)$ & $95.7(12.9)$ & $.81(.59-.92)$ & 5.40 & 15.0 \\
\hline Right LE reaction time, $\mathrm{s}$ & $0.55(0.1)$ & $0.519(0.1)$ & $.92(.81-.97)$ & 0.02 & 0.07 \\
\hline Right LE hits, $\mathrm{n}$ & $94.0(11.4)$ & $97.9(15.0)$ & $.83(.61-.93)$ & 5.5 & 15.25 \\
\hline
\end{tabular}

Abbreviations: CI, confidence interval; ICC, intraclass correlation coefficient; LE, lower-extremity; MDC, minimal detectable change; UE, upper-extremity. Note: Data are presented as mean (SD). 
This study is not without limitations. First, participants could have had a history of injury but were not excluded unless their injury was sustained within 12 months before testing. Although participants were considered healthy at the time of testing, we cannot conclude these results are representative of an injury-free population. Second, the upper-extremity task required the use of both arms, while the lowerextremity task required the use of one limb at a time. This was justified from a neurophysiology perspective. ${ }^{11}$ Changes in brain structure and function, specifically in the motor cortex, can be altered by the type of injury sustained and the particular mechanism of injury. ${ }^{11}$ Type of injury (eg, concussion, anterior cruciate ligament tear) could influence how clinicians implement these tasks, and the creation of a single-arm protocol or double-leg protocol may be warranted. Third, we did not complete an interrater reliability assessment of the raters. However, each rater was trained on the same standard operating procedures prior to testing, and the procedures were followed for each testing session. Finally, we did not control for upper-extremity injury within the last 12 months. Future research should consider expanding the injury-history screening.

The upper- and lower-extremity VMRT tasks using the FitLight system demonstrated suitable reliability for assessing upper- and lower-extremity VMRT. The MDC values calculated provide a mechanism to interpret the effectiveness of VMRT interventions. Future studies should explore the validity of the upper- and lower-extremity tasks, and identify the most effective use of the tasks for assessment and rehabilitation purposes in specific clinical populations.

\section{References}

1. Miller JL, Low K. Motor processes in simple, go/no-go, and choice reaction time tasks: a psychophysiological analysis. J Esp Psychol Hum Percept Perform. 2001;27(2):266-289. PubMed ID: 11318047

2. Wilkerson GB, Simpson KA, Clark RA. Assessment and training of visuomotor reaction time for football injury prevention. J Sport
Rehabil. 2017;26(1):26-34. PubMed ID: 27632871 doi:10.1123/jsr. 2015-0068

3. Picha K, Quintana C, Glueck A, Hoch M, Heebner NR, Abt JP. Reliability of 5 novel reaction time and cognitive load protocols. $J$ Sport Rehabil. 2018;27(5):1-4. PubMed ID: 29809102 doi:10.1123/ jsr.2018-0042

4. Wells AJ, Hoffman JR, Beyer KS, et al. Reliability of the dynavi$\operatorname{sion}^{\mathrm{TM}} \mathrm{d} 2$ for assessing reaction time performance. J Sports Sci Med. 2014;13(1):145-150. PubMed ID: 24570618

5. Swanik C, Covassin T, Stearne DJ, Schatz P. The relationship between neurocognitive function and noncontact anterior cruciate ligament injuries. Am J Sports Med. 2007;35(6):943-948. PubMed ID: 17369562 doi:10.1177/0363546507299532

6. FitLight Trainer. FITLIGHT Corp. 2019. https://www.fitlight training.com/products/. Accessed April 5, 2020.

7. Bujang M.A., Baharum N. A simplified guide to determination of sample size requirements for estimating the value of intraclass correlation coefficient: a review. Achr Orogac Sci. 2017;12(1): $1-11$.

8. Millikan N, Grooms DR, Hoffman B, Simon JE. The development and reliability of four clinical neurocognitive single-leg hop tests: implications for return to activity decision making. J Sport Rehabil. 2019:28(5):536-544. PubMed ID: 30426885 doi:10.1123/jsr.20180037

9. Zwierko T, Florkiewicz B, Fogtman S, Kszak-Krzyanowska A. The ability to maintain attention during visuomotor task performance in handball players and non-athletes. Cent Eur J Sports Sci Med. 2014;3(7):99-106.

10. Koo TK, Li MY. A guideline of selecting and reporting intraclass correlation coefficients for reliability research. J Chiropr Med. 2016; 15(2):155-163. PubMed ID: 27330520 doi:10.1016/j.jcm.2016. 02.012

11. Grooms DR, Page SJ, Nichols-Larsen DS, Chaudhari AM, White SE, Onate JA. Neuroplasticity associated with anterior cruciate ligament reconstruction. J Orthop Sports Phys Ther. 2017;47(3):180-189. PubMed ID: 27817301 doi:10.2519/jospt.2017.7003 